\title{
Imperfect nesting and scattering due to disorder in spin-density-waves
}

\author{
X.Z. HUANG and K. MAKI*
}

T-11, MS-B262, Los Alamos National Labs., Los Alamos, NM 87545, U.S.A.

* Department of Physics, University of Southern California, Los Angeles, CA 90089-0484, U.S.A.

As is well-known both imperfect nesting and scattering reduces the spin-density-wave transition temperature and the quasi-particle energy gap at $T=0 K$. We extend our earlier analysis to the condensate density $f_{1}$ and the pinning potential thus the threshold field, at both $T=0 K$ and $T=T_{c}$ when both imperfect nesting and scattering effect are present. At $T=0 K, f_{1}=1$ in the presence of both imperfect nesting and scattering until the quasi-particle energy gap is closed. Consequently, the threshold field $E_{T}$ is almost constant until SDW is almost destroyed, where $E_{T}$ suddenly moves up. We found that $E_{T}$ exhibits a small peak just before $T_{c}$ for small scattering rates. This result may help disentangle the mechanism behind the rapid cooling of (TMTSF) ${ }_{2} \mathrm{ClO}_{4}$.

Extending earlier models by Horovitz et al. [1] and Yamaji [2] we have shown recently [3] that the model with imperfect nesting can account for a number of features associated with SDW in Bechgaard salts like (TMTSF) ${ }_{2} \mathrm{PF}_{6}$ etc. and CDW's in $\mathrm{NbSe}_{3}$ within mean field theory. For example the large ratio $\Delta_{a} / T_{c}$ observed in CDW's of $\mathrm{NbSe}_{3}$ is accounted for in terms of imperfect nesting contrary to the prevailing but incorrect view based on the large fluctuation or strong coupling [4]. Here $\Delta_{a}$ is the apparent energy gap deduced from the activation energy of the electric resistance.

The impurity scattering is unavoidable in SDW (or CDW). Further in (TMTSF) ${ }_{2} \mathrm{ClO}_{4}$ the quenching of the anion disorder by rapid cooling will probably introduce an additional scattering due to the disorder for example. In this paper we discuss the threshold electric field when both imperfect nesting and scattering effect are present.

We limit ourselves to SDW for simplicity. - the results obtained here also apply to the quasione-dimensional CDW. We shall start with the Hamiltonian first introduced by Yamaji [2]:

$$
H=\sum_{p, \alpha} \epsilon(p) c_{p \alpha}^{\dagger} c_{p a}+U \sum_{q} n_{q \uparrow} n_{-q \downarrow}
$$

with the quasiparticle energy:

$$
\epsilon(p)=v\left(p_{1}-p_{F}\right)-2 t_{b} \cos \left(b p_{2}\right)-\varepsilon_{0} \cos \left(2 b p_{2}\right)
$$

Here $c_{p \alpha}^{\dagger}$ and $c_{p \alpha}$ are the creation and annihilation operator of electrons with momentum $p$ and $\operatorname{spin} \alpha$, and $n_{q \dagger}$ and $n_{-q \downarrow}$ are the corresponding number operators. $\varepsilon_{0}$ is the parameter characterizing the imperfect nesting effect which is associated with the quasi-one dimensionality.

Within the mean field approximation, the quasiparticle Green's function in the spinor representation is given by:

$$
G^{-1}\left(p, \omega_{n}\right)=i \omega_{n}-\varepsilon_{0} \cos \left(2 b p_{2}\right)-\xi \rho_{3}-\Delta \rho_{1} \sigma_{3}
$$

where $\rho_{1}$ and $\rho_{3}$ are Pauli spin matrices operating on the spinor space formed by the left- and right-going electrons, $\omega_{n}$ is the Matsubara frequency, and

$$
\xi=v\left(p_{1}-p_{F}\right)-2 t_{b} \cos \left(b p_{2}\right)
$$

The order parameter is determined self-consistently by

$$
\Delta=U T \sum_{n} \int \frac{d^{3} p}{(2 \pi)^{3}} \operatorname{Tr}\left[\rho_{1} G\left(p, \omega_{n}\right)\right]
$$


We add the impurity scattering effect into the above model. Following the standard diagram approach [5], the impurity effect can be incorporated by replacing $\omega_{n}$ and $\Delta$ in Eq. (3) by $\bar{\omega}_{n}$ and $\tilde{\Delta}$

$$
\tilde{\omega}_{n}=\omega_{n}+\frac{1}{2}\left(\Gamma_{1}+\Gamma_{2}\right)\left\langle\frac{\left(\tilde{\omega}_{n}+i \varepsilon_{0} \cos (\chi)\right)}{\sqrt{\left(\tilde{\omega}_{n}+i \varepsilon_{0} \cos (\chi)\right)^{2}+\tilde{\Delta}^{2}}}\right\rangle
$$

and

$$
\tilde{\Delta}=\Delta-\frac{1}{2} \Gamma_{1}\left\langle\frac{\tilde{\Delta}}{\sqrt{\left(\tilde{\omega}_{n}+i \varepsilon_{0} \cos (\chi)\right)^{2}+\tilde{\Delta}^{2}}}\right\rangle
$$

where $\Gamma_{1}$ and $\Gamma_{2}$ are the forward and backward scattering rate respectively. $\chi=2 b p_{2}$ is the angle related to the second direction, and $\langle\cdots\rangle$ denotes the angular average over $\chi$.

The self-consistent equation Eq. (5) can be rewritten by substituting Eqs. (6) and (7) as:

$$
\lambda^{-1}=2 \pi T \sum_{n=0}^{n_{c}} \frac{\tilde{\Delta}}{\Delta}\left\langle\frac{1}{\sqrt{\left(\tilde{\omega}_{n}+i \varepsilon_{0} \cos (\chi)\right)^{2}+\tilde{\Delta}^{2}}}\right\rangle
$$

where

$$
\lambda=U N_{0}
$$

is the dimensionless coupling constant, and $N_{0}$ is the density of states at the Fermi surface.

We concentrate on calculating the threshold electric field $E_{T}$. In the strong pinning limit, the threshold field $E_{T}^{s}$ is the ratio of the pining potential $F$ and the condensate density $f_{1}$ [3]:

$$
E_{T}^{s}=\frac{F}{f_{1}}
$$

and in the 3-D weak pinning limit,

$$
E_{T}^{w} \propto\left(E_{T}^{s}\right)^{4}
$$

We compute $E_{T}^{s}$ in the following and denote it simply by $E_{T}$.

In our earlier analysis [3], we calculated $E_{T}$ as a function of temperature when only imperfect nesting $\varepsilon_{0}$ is present. We found that when $T$ gets close to $T_{c}\left(\varepsilon_{0}\right), E_{T}$ moves up as $\varepsilon_{0}$ increases. In the presence of both imperfect nesting and scattering effect, $F$ is given by:

$$
F=2 \pi T \sum_{n=0}^{\infty} \tilde{\Delta}^{2}\left\langle\left[\left(\tilde{\omega}_{n}+i \eta\right)^{2}+\tilde{\Delta}^{2}\right]^{-\frac{1}{2}}\right\rangle^{2}
$$

and $f_{1}$ is given by:

$$
f_{1}=\frac{2 \pi T}{\Delta} \sum_{n=0}^{\infty}\left(\left\langle\frac{\tilde{\Delta}^{3}}{R^{3}}\right\rangle+\frac{\Gamma_{1}}{2} \tilde{\Delta}^{3} \Xi\right)\left(1-\frac{\Gamma}{\Delta}\left\langle\frac{\tilde{\Delta}^{3}}{R^{3}}\right\rangle-\frac{\Gamma_{1}}{4} \Delta\left(\Gamma_{1}+\Gamma_{2}\right) \tilde{\Delta}^{3} \Xi\right)^{-1},
$$

where

$$
R=\sqrt{\left(\tilde{\omega}_{n}+i \eta\right)^{2}+\tilde{\Delta}^{2}}
$$

and

$$
\Xi=\left\langle\frac{1}{R^{3}}\right\rangle\left\langle\frac{\left(\tilde{\omega}_{n}+i \eta\right)^{2}}{R^{3}}\right\rangle-\left\langle\frac{\left(\tilde{\omega}_{n}+i \eta\right)}{R^{3}}\right\rangle^{2} .
$$

In order to understand the different role of the imperfect nesting $\varepsilon_{0}$ and the scattering $\Gamma$ on $E_{T}$, we first calculate the temperature dependence of $E_{T}$ when only $\Gamma$ exists, i.e., $\varepsilon_{0}=0$. In this limit, $F$ and $f_{1}$ are given respectively by:

$$
F=2 \pi T \sum_{n=0}^{\infty} \frac{1}{u_{n}^{2}+1}
$$




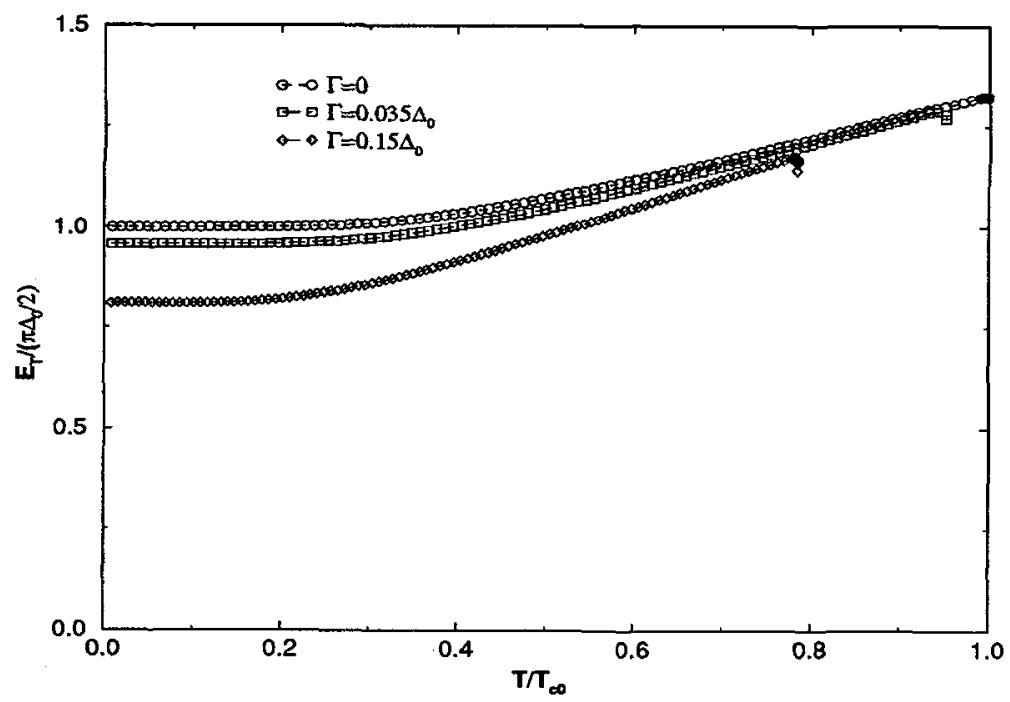

Figure 1: Threshold field $E_{T}$ as function of temperature $T$ for several $\Gamma$ when $\varepsilon_{0}=0:(\circ) \Gamma=0$; () $\Gamma=0.035 \Delta_{0} ;(\diamond) \Gamma=0.15 \Delta_{0} . \Delta_{0}$ is the order parameter when $\Gamma=\varepsilon=T=0$. $E_{T}$ is normalized by $\pi \Delta_{s} / 2$ the $E_{T}$ value when $T=0, \Gamma=0$.

$$
f_{1}=\frac{2 \pi T}{\Delta} \sum_{n=0}^{\infty} \frac{1}{\left(u_{n}^{2}+1\right)^{\frac{3}{2}}}\left[1-\frac{\Gamma}{\Delta} \frac{1}{\left(u_{n}^{2}+1\right)^{\frac{3}{2}}}\right]^{-1},
$$

where $u_{n}$ is obtained by solving:

$$
\frac{\omega_{n}}{\Delta}=u_{n}\left(1-\frac{\Gamma}{\Delta}\left(1+u_{n}^{2}\right)^{-\frac{2}{2}}\right)
$$

The order parameter $\Delta$ is obtained by:

$$
\begin{aligned}
& -\ln \left(\frac{\Delta(T, \Gamma)}{\Delta_{0}}\right)= \begin{cases}\frac{\pi}{4} \zeta & (\zeta \leq 1) \\
\operatorname{arccosh}(\zeta)+\frac{1}{2}\left(\zeta \arcsin \left(\zeta^{-1}\right)-\sqrt{1-\zeta^{-2}}\right) & (\zeta>1)\end{cases} \\
& +2 \int_{u_{0}}^{\infty} \frac{d E}{\Delta} \Re \mathrm{e}\left(\frac{1}{\sqrt{u^{2}-1}}\right) f(\beta E) \text {, }
\end{aligned}
$$

with

$$
\zeta=\frac{\Gamma}{\Delta}
$$

At $T=0 K$, it can be shown that:

$$
f_{1}= \begin{cases}1 & \text { for } \zeta \leq 1 \\ 1-\sqrt{1-\zeta^{-2}} & \text { for } \zeta>1\end{cases}
$$

and

$$
F=\Delta \begin{cases}\frac{\pi}{2}-\frac{2}{3} \zeta & \text { for } \zeta \leq 1 \\ \frac{\pi}{2}-\arccos \zeta^{-1}-\frac{\zeta}{3}\left(2-\left(2+\zeta^{-2}\right) \sqrt{1-\zeta^{-2}}\right) & \text { for } \zeta>1\end{cases}
$$

A reference value of $E_{T}$ at $\Gamma=0$ can be calculated as

$$
E_{T}^{0}=E_{T}\left(T=0, \varepsilon / \Delta_{0}=0, \Gamma / \Delta_{0}\right)=\frac{\pi}{2} \Delta_{0}
$$




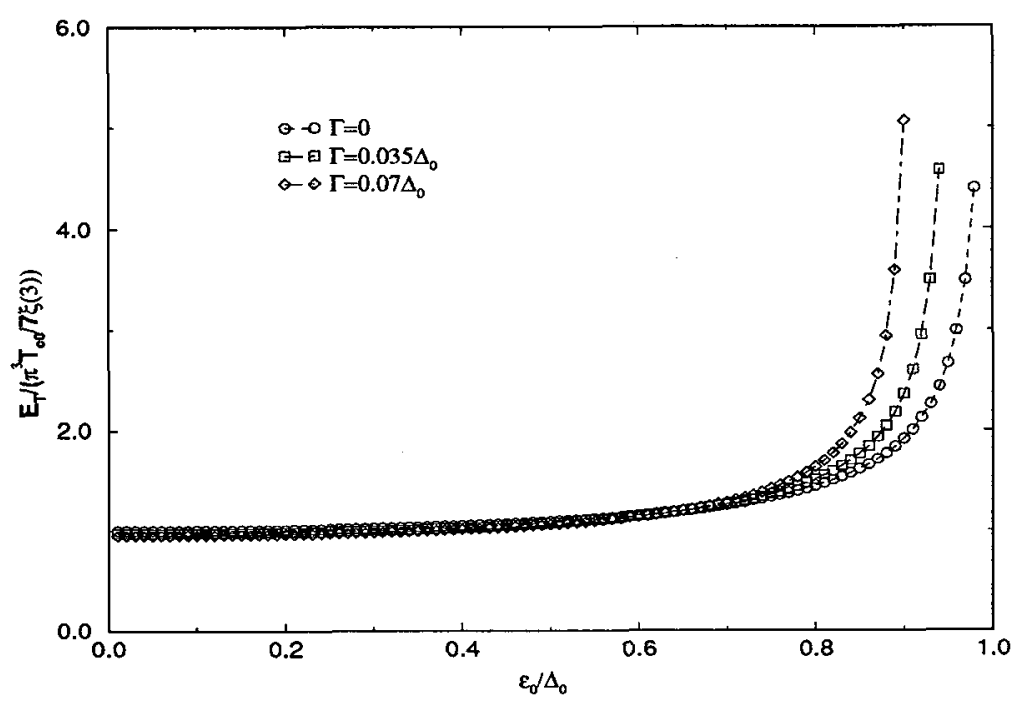

Figure 2: Threshold field $E_{T}$ as function of $\varepsilon_{0}$ at $T=T_{c}$ for several $\Gamma(0) \Gamma=0 ;(\square) \Gamma=0.035 \Delta_{0} ;(\diamond)$ $\Gamma=0.07 \Delta_{0} . \Gamma_{2}=\frac{1}{2} \Gamma_{1}$ is chosen. $E_{T}$ is normalized by $\pi^{3} T_{c 0} / 7 \zeta(3)$ the $E_{T}$ value when $T=T_{c}, \Gamma=0$.

Near $T=T_{c}$, where $T_{c}$ is determined by:

$$
\ln \left(\frac{T_{c 0}}{T_{c}}\right)=\psi\left(\frac{1}{2}+\frac{\Gamma}{2 \pi T_{c}}\right)-\psi\left(\frac{1}{2}\right)
$$

with $\psi$ the Digamma function, $E_{T}$ is given by:

$E_{T}=\frac{\pi^{3} T}{7 \zeta(3)}\left\{1+\left(\frac{\pi^{4}}{14 \zeta(3)}-\frac{28 \zeta(3)}{\pi^{2}}\right) \frac{\Gamma}{2 \pi T}+\left(\frac{93}{14} \frac{\zeta(5)}{\zeta(3)}-\frac{\pi^{2}}{3}+\left(310 \zeta(5) \pi^{-2}-\frac{17 \pi^{6}}{210 \zeta(3)}\right) \frac{\Gamma}{2 \pi T}\right)\left(\frac{\Delta}{2 \pi T}\right)^{2}\right\}$.

From the above equation, one expects that $E_{T}$ has a small peak when $T$ gets very close to $T_{c}$ for small $\Gamma$ since the coefficient in front of $\Delta^{2}$ would be positive. At exactly $T=T_{c}$, for small $\Gamma$,

$$
E_{T}=\frac{\pi^{3} T_{c 0}}{7 \zeta(3)}\left(1+\left(\frac{\pi^{4}}{14 \zeta(3)}-\frac{28 \zeta(3)}{\pi^{2}}-\frac{\pi^{2}}{2}\right) \frac{\Gamma}{2 \pi T_{c 0}}\right)
$$

The coefficient before $\Gamma$ is negative, therefor one expects that for $\varepsilon_{0}=0$, at both $T=0 K$ (see Eqs. 22 and 21) and $T=T_{c}, E_{T}$ decreases as $\Gamma$ increases (see also Fig. 2 below). Fig. 1 shows $E_{T}$ as function of $T$ for several $\Gamma$. One clearly sees the small peak near $T_{c}$.

Including both imperfect nesting $\varepsilon_{0}$ and scattering rate $\Gamma$ increases the complexity of the numerical computation. Instead of computing $E_{T}$ for the whole range of temperatures from $0 K$ to $T_{c}(\varepsilon, \Gamma)$, we calculated $E_{T}$ at $T=0 K$ and $T=T_{c}$. For $T=T_{c}$, analytical result exists, and Fig. 2 shows the result of $E_{T}$ at $T=T_{c}$ for several $\Gamma . F$ and $f_{1}$ are given by:

$$
\begin{gathered}
F=\Delta^{2} 2 \pi T_{c} \sum_{n=0}^{\infty} \frac{1}{\left(\sqrt{\left.\omega_{n}+\frac{\Gamma_{1}+\Gamma_{2}}{2}\right)^{2}+\varepsilon_{0}^{2}}+\frac{\Gamma_{1}}{2}\right)^{2}} \\
f_{1}=\Delta^{2} \pi T_{c} \sum_{n=0}^{\infty} \frac{1}{\left(\sqrt{\left(\omega_{n}+\frac{\Gamma_{1}+\Gamma_{2}}{2}\right)^{2}+\varepsilon_{0}^{2}}+\frac{\Gamma_{1}}{2}\right)^{3}}\left\{\frac{2\left(\omega_{n}+\frac{\Gamma_{1}+\Gamma_{2}}{2}\right)^{2}-\varepsilon_{0}^{2}}{\left(\omega_{n}+\frac{\Gamma_{1}+\Gamma_{2}}{2}\right)^{2}+\varepsilon_{0}^{2}}-\frac{\Gamma_{1}}{2} \frac{\varepsilon_{0}^{2}}{\left(\left(\omega_{n}+\frac{\Gamma_{1}+\Gamma_{2}}{2}\right)^{2}+\varepsilon_{0}^{2}\right)^{\frac{3}{2}}}\right\}
\end{gathered}
$$

On the other hand, no analytical expression exists at $T=0 K$ for general $\varepsilon_{0}$ and $\Gamma$, other than changing the summation over $\omega_{n}$ in the expressions of both $F$ and $f_{1}$ into integration over continuous 


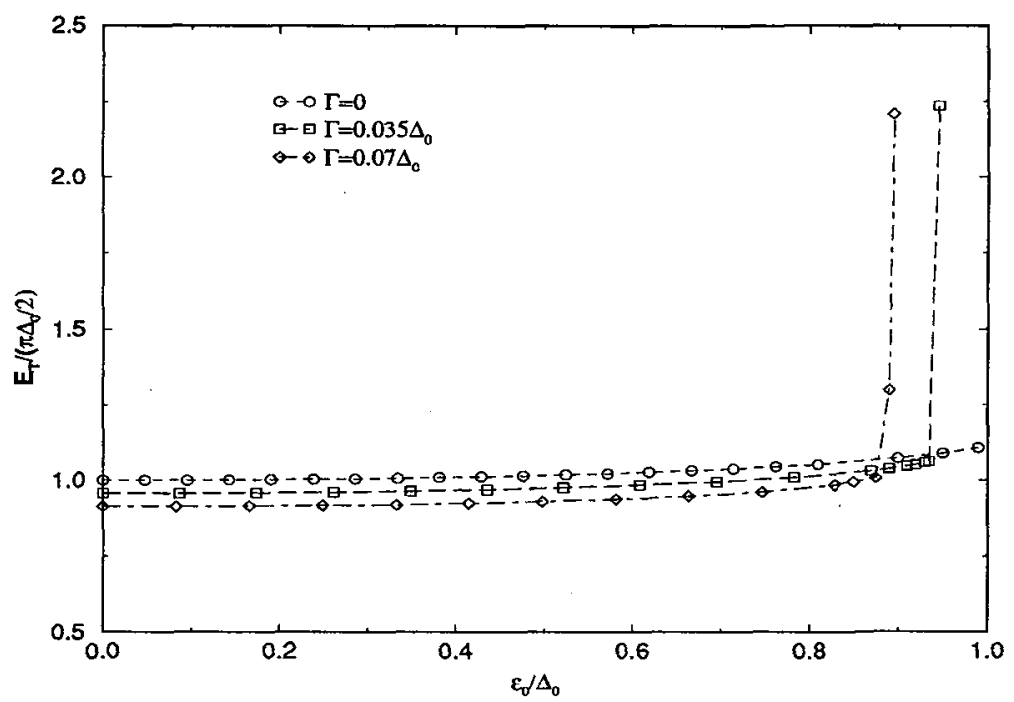

Figure 3: Threshold field $E_{T}$ as function of $\varepsilon_{0}$ at $T=0 K$ for several $\Gamma$ (o) $\Gamma=0$; (ㅁ) $\Gamma=0.035 \Delta_{0}$; $(\diamond) \Gamma=0.07 \Delta_{0} . \Gamma_{2}=\frac{1}{2} \Gamma_{1}$ is chosen. $E_{T}$ is normalized by $\pi \Delta_{s} / 2$ the $E_{T}$ value when $T=0, \Gamma=0$.

variable $\omega$. Fig. 3 shows the result of $E_{T}$ for several $\Gamma$. One should notice that as $\varepsilon_{0}$ and $\Gamma$ both come to play a role, $f_{1}$ is identically equal to unity until the quasi-particle energy gap is closed. It is similar to the case when $\Delta$ becomes smaller than $\Gamma$ in the absence of $\varepsilon_{0}$, but is more complicated when $\varepsilon_{0} \neq 0$. This is reflected in the sudden move-up in Fig. 3 as $\varepsilon_{0}$ becomes closer to its critical value of completely suppressing $T_{c}$. From Figs. 2 and 3 , one notices that at both $T=0 K$ and $T=T_{c}, E_{T}$ first decreases with $\Gamma$ for small $\varepsilon_{0}$, but later increases with $\Gamma$ as $\varepsilon_{0}$ increases.

In conclusion, we have analyzed the impurity scattering in SDW (or CDW) with imperfect nesting. For all $\varepsilon_{0}$ the impurity scattering suppresses the order parameter, though the effect is more significant for larger $\varepsilon_{0}$. On the other hand the effect on the density of states becomes less visible as $\varepsilon_{0}$ increases. The threshold filed $E_{T}$ in general is a complicated function of temperature when both $\varepsilon_{0}$ and $\Gamma$ are present. $\epsilon_{0}$ plays different role than $\Gamma$ in changing $E_{T}$. The small peak near $T_{c}$ may explain a recent experimental result obtained by Nomura et al. [6].

This work was supported by the National Science Foundation under grant No. DMR89-15285 and the U.S. Department of Energy.

\section{REFERENCES}

[1] Horovitz B., Weger M. and Gutfreund H., Phys. Rev. B. 9 (1974) 1246; Horovitz B., Gutfreund H. and Weger M., ibid. 12 (1975) 3174.

[2] Yamaji K., J. Phys. Soc. Jpn. 51 (1982) 2787;

[3] Huang X. and Maki K., Phys. Rev. B. 40 (1989) 2575; ibid. 42 (1990) 6489; 46 (1992) 162; Synthetic Metals 41-43 (1991) 3827;ibid. 56 (1993) 2767.

[4] Monceau P. in Electric Properties of Inorganic Quasi-One Dimensional Materials edited by Monceau P. (Reidel, Dordrecht 1985) p. 139.

[5] Abrikosov A. A. and Gor'kov L. P., JETP 12 (1961) 1243.

[6] Nomura, et al., preprint. 